\title{
Construction method of the cost function for the minimax shape optimization problem
}

\author{
Kouhei Shintani ${ }^{1}$ and Hideyuki Azegami ${ }^{1}$ \\ ${ }^{1}$ Nagoya University, A4-2 (780) Furo-cho, Chikusa-ku, Nagoya 464-8601, Japan \\ E-mailshintani@az.cs.is.nagoya-u.ac.jp
}

Received April 1, 2013, Accepted June 7, 2013

\begin{abstract}
The present paper describes a method by which to formulate a shape optimization problem of a linear elastic continuum for minimizing the maximum value of a strength measure, such as the von Mises stress. In order to avoid the irregularity of the shape derivative of the maximum value, the Kreisselmeier-Steinhauser function of the strength measure is used as the cost function. In the cost function, a parameter is used to control the regularity of the shape derivative. In the present paper, we propose a rule by which to appropriately determine the parameter. The effectiveness of the proposed rule is confirmed through a numerical example of a cantilever problem.
\end{abstract}

Keywords calculus of variations, boundary value problem, shape optimization, H1 gradient method, Kreisselmeier-Steinhauser function

Research Activity Group Mathematical Design

\section{Introduction}

In the design of parts of machines or mechanical structures, strength is one of the most important factors. In practice, the maximum von Mises stress is evaluated at all times and all locations for parts constructed from ductile metal, which is modeled as a linear elastic body, and is used as a measure of the strength. Hence, in order to find the optimum shape of a linear elastic body while maximizing the strength, we must construct a shape optimization problem involving the minimization of the maximum von Mises stress, which we refer to as the minimax shape optimization problem. However, this problem is irregular because we cannot define the Fréchet derivative with respect to domain variation, which we refer to as the shape derivative, of the cost function, such as the maximum value of the von Mises stress.

In order to avoid irregularity, the KreisselmeierSteinhauser function [1], which is referred to hereinafter as the KS function, with respect to a strength measure such as the von Mises stress has been used instead of the maximum value of a strength measure. We can define the shape derivative of the KS function and obtain a numerical solution by the finite element method, as shown in [2].

However, in the KS function, a parameter $p \in(0, \infty)$ is used to control the regularity of the shape derivative of the KS function. When $p \rightarrow \infty$, the value of the KS function approaches the maximum value of the strength measure, while the regularity worsens simultaneously. Actually, convergence phenomena are affected by the value of $p$. An appropriate value of $p$ has been determined empirically.

In the present paper, we propose a rule by which to determine the value of $p$. Using this rule, we construct a shape optimization problem with the KS function as a cost function. The method for computing the shape derivative of the cost function is given as an adjoint variable method considering the rule for $p$. A solution to this new problem is presented based on the algorithm of sequential quadratic programming using the $H^{1}$ gradient method for reshaping in order to maintain the smoothness of the boundary [3-6]. The effectiveness of the rule for $p$ is confirmed based on a numerical solution to a cantilever problem.

\section{Admissible set of design variables}

First, let us define the admissible set of design variable for the shape optimization problem. Let $D_{0}$ and $\Omega_{0} \subset D_{0}$ be $d \in\{2,3\}$ dimensional domains with Lipschitz boundaries, which are denoted by $\partial D_{0}$ and $\partial \Omega_{0}$. On $\partial \Omega_{0}, \Gamma_{\mathrm{D} 0} \subset \partial \Omega_{0}$ and $\Gamma_{\mathrm{N} 0}=\partial \Omega_{0} \backslash \bar{\Gamma}_{\mathrm{D} 0}\left(\bar{\Gamma}_{\mathrm{D} 0}=\right.$ $\left.\Gamma_{\mathrm{D} 0} \cup \partial \Gamma_{\mathrm{D} 0}\right)$ denote the Dirichlet boundary and the Neumann boundary, respectively. Moreover, let $\Gamma_{p 0} \subset \Gamma_{\mathrm{N} 0}$ be a non-homogeneous Neumann boundary. We assume that $D_{0}$ and $\Omega_{0}$ are fixed and that mapping $\phi: D_{0} \rightarrow \mathbb{R}^{d}$ included in a Banach of

$$
Y=W^{1, \infty}\left(D_{0} ; \mathbb{R}^{d}\right)
$$

is a design variable in the shape optimization problem. Moreover, we let

$$
\begin{gathered}
\mathcal{D}=\left\{\phi \in Y \mid\left\|\phi-\phi_{0}\right\|_{W^{1, \infty}\left(D_{0} ; \mathbb{R}^{d}\right)}<1, \phi\left(\Omega_{0}\right) \subseteq D_{0}\right. \\
\text { piecewise } C^{2} \text { class on } \Gamma_{p 0} \\
\left.\phi=\phi_{0} \text { on } \Gamma_{\mathrm{C} 0}\right\}
\end{gathered}
$$

be the admissible set of design variable $\phi$, where $\Gamma_{\mathrm{C} 0} \subset$ $\partial \Omega_{0}$ denotes a boundary on which domain variation is constrained due to design considerations. In (2), $\left\|\phi-\phi_{0}\right\|_{W^{1, \infty}\left(D_{0} ; \mathbb{R}^{d}\right)}<1$ is used to assure one-to-one 


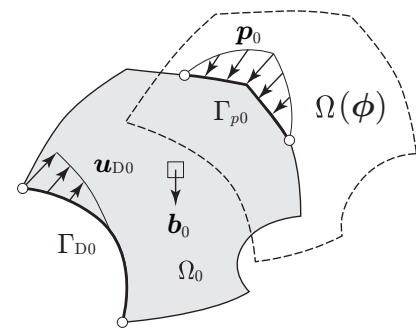

Fig. 1. Initial domain $\Omega_{0}$ and variation domain $\Omega(\phi)$.

mapping. In the present paper, we use the notation $\Omega(\phi)$ for $\left\{\phi(\boldsymbol{x}) \mid \boldsymbol{x} \in \Omega_{0}\right\}$ and $\partial \Omega^{-}(\phi)$ for the open set $\partial \Omega(\phi) \backslash \Theta(\phi)$, where $\Theta(\phi)$ denotes the set of corner points of measure 0 .

\section{Main problem}

Using the design variable $\phi \in \mathcal{D}$, let us define the main problem as a shape optimization problem. In the present paper, we consider a linear elastic problem as the main problem. We have the following assumptions.

(H1) For $q>d, \boldsymbol{b} \in L^{2 q}\left(D_{0} ; \mathbb{R}^{d}\right), \boldsymbol{p} \in W^{1,2 q}$ $\left(D_{0} ; \mathbb{R}^{d}\right), \boldsymbol{u}_{\mathrm{D}} \in W^{1,2 q}\left(D_{0} ; \mathbb{R}^{d}\right)$, and $\boldsymbol{C} \in W^{1, \infty}$ $\left(D_{0} ; \mathbb{R}^{d \times d \times d \times d}\right)$ denote the volume force, the traction force, and the given displacement and stiffness, respectively. For $\boldsymbol{C}$, we assume that there exist positive constants $\alpha$ and $\beta$ such that $\boldsymbol{X} \cdot(\boldsymbol{C} \boldsymbol{X}) \geq$ $\alpha\|\boldsymbol{X}\|^{2},|\boldsymbol{X} \cdot(\boldsymbol{C} \boldsymbol{Y})| \leq \beta\|\boldsymbol{X}\|\|\boldsymbol{Y}\|$ for all $\boldsymbol{X}, \boldsymbol{Y} \in$ $\left\{\boldsymbol{X} \in \mathbb{R}^{d \times d} \mid \boldsymbol{X}=\boldsymbol{X}^{\mathrm{T}}\right\}$ and $C_{i j k l}=C_{k l i j}$ at almost all of $D_{0}$.

(H2) For $\partial \Omega(\phi)$, opening angles at corner points $\Theta(\phi)$ and the boundary of mixed boundary conditions $\partial \Gamma_{\mathrm{D}}(\phi)$ are less than $\pi$ and $\pi / 2$, respectively (see Fig. 1).

Under the assumptions, we let

$$
\mathcal{S}=\left\{\boldsymbol{u} \in W^{2,2 q}\left(D_{0} ; \mathbb{R}^{d}\right) \mid \boldsymbol{\phi} \in \mathcal{D}\right\}
$$

be the admissible set for displacement $\boldsymbol{u}$. Let $\boldsymbol{T}(\boldsymbol{u})=$ $\boldsymbol{C E}(\boldsymbol{u})$ and $\boldsymbol{E}(\boldsymbol{u})=\left[\boldsymbol{\nabla} \boldsymbol{u}^{\mathrm{T}}+\left(\boldsymbol{\nabla} \boldsymbol{u}^{\mathrm{T}}\right)^{\mathrm{T}}\right] / 2$ denote the stress and strain, respectively. Moreover, we use $\boldsymbol{\nu}$ as the outer unit normal on the boundary.

Problem 1 (Linear elastic problem) For $\phi \in \mathcal{D}$, let $(\mathrm{H} 1)$ and $(\mathrm{H} 2)$ be satisfied. Find $\boldsymbol{u} \in \mathcal{S}$ such that

$$
\begin{aligned}
& -\nabla^{\mathrm{T}} \boldsymbol{T}(\boldsymbol{u})=\boldsymbol{b}^{\mathrm{T}} \text { in } \Omega(\phi), \\
& \boldsymbol{T}(\boldsymbol{u}) \boldsymbol{\nu}=\boldsymbol{p} \text { on } \Gamma_{p}^{-}(\boldsymbol{\phi}), \\
& \boldsymbol{T}(\boldsymbol{u}) \boldsymbol{\nu}=\mathbf{0} \text { on } \Gamma_{\mathrm{N}}^{-}(\boldsymbol{\phi}) \backslash \bar{\Gamma}_{p}(\boldsymbol{\phi}), \\
& \boldsymbol{u}=\boldsymbol{u}_{\mathrm{D}} \text { on } \Gamma_{\mathrm{D}}(\boldsymbol{\phi}) .
\end{aligned}
$$

For use in Section 5, we here define the Lagrange function for the main problem (Problem 1) as

$$
\begin{aligned}
& \mathscr{L}_{\mathrm{M}}(\boldsymbol{\phi}, \boldsymbol{u}, \boldsymbol{v}) \\
& =\int_{\Omega(\phi)}(-\boldsymbol{T}(\boldsymbol{u}) \cdot \boldsymbol{E}(\boldsymbol{v})+\boldsymbol{b} \cdot \boldsymbol{v}) \mathrm{d} x+\int_{\Gamma_{\mathrm{N}}(\boldsymbol{\phi})}^{\boldsymbol{p} \cdot \boldsymbol{v} \mathrm{d} \gamma} \\
& \quad+\int_{\Gamma_{\mathrm{D}}^{-}(\phi)}\left[\left(\boldsymbol{u}-\boldsymbol{u}_{\mathrm{D}}\right) \cdot \boldsymbol{T}(\boldsymbol{v}) \boldsymbol{\nu}+\boldsymbol{v} \cdot \boldsymbol{T}(\boldsymbol{u}) \boldsymbol{\nu}\right] \mathrm{d} \gamma
\end{aligned}
$$

where $\boldsymbol{v} \in \mathcal{S}$ is introduced as the Lagrange multiplier. With $\mathscr{L}_{\mathrm{M}}(\boldsymbol{\phi}, \boldsymbol{u}, \boldsymbol{v})$, if $\boldsymbol{u}$ is the solution of Problem 1, then

$$
\mathscr{L}_{\mathrm{M}}(\boldsymbol{\phi}, \boldsymbol{u}, \boldsymbol{v})=0
$$

holds for all $\boldsymbol{v} \in \mathcal{S}$.

\section{Shape optimization problem}

Using the solution $\boldsymbol{u}$ of Problem 1 for $\phi \in \mathcal{D}$, we define the cost functions as follows. In the present paper, we are attempting to construct a cost function for the strength of a linear elastic body. Here, we let $\sigma: \mathbb{R}^{d} \rightarrow \mathbb{R}$ be a function of $\boldsymbol{u}$ representing a measure of strength. In the present paper, we use the von Mises stress for the measure given by

$$
\sigma(\boldsymbol{u})=\sqrt{\frac{3}{2} \boldsymbol{T}_{\mathrm{D}}(\boldsymbol{u}) \cdot \boldsymbol{T}_{\mathrm{D}}(\boldsymbol{u})},
$$

where $\boldsymbol{T}_{\mathrm{D}}(\boldsymbol{u})$ denotes the deviator stress, which is defined as

$$
\boldsymbol{T}_{\mathrm{D}}(\boldsymbol{u})=\boldsymbol{T}(\boldsymbol{u})-\frac{1}{3} \operatorname{tr}(\boldsymbol{T}(\boldsymbol{u})) \boldsymbol{I},
$$

and $\boldsymbol{I}$ denotes the unit tensor.

As a cost function of strength, we set the objective function as follows:

$$
f_{0}(\boldsymbol{\phi}, \boldsymbol{u}, p)=\frac{1}{p} \ln \left(\frac{\int_{\Omega(\boldsymbol{\phi})} \mathrm{e}^{p \sigma(\boldsymbol{u})} \mathrm{d} x}{\int_{\Omega(\boldsymbol{\phi})} \mathrm{d} x}\right) .
$$

In $(5), p \in(0, \infty)$ is assumed to be a constant in the previous papers $[1,2]$. However, in the present paper, we assume that

$$
p=\frac{\int_{\Omega(\phi)} \mathrm{d} x}{\int_{\Omega(\boldsymbol{\phi})} \sigma(\boldsymbol{u}) \mathrm{d} x}-\frac{1}{c_{0}}
$$

holds, where $c_{0}$ denotes a positive constant, which indicates $\max _{\boldsymbol{x} \in \Omega_{0}} \sigma(\boldsymbol{u}(\boldsymbol{x}))$ with the solution $\boldsymbol{u}$ of Problem 1 for $\Omega_{0}$. Moreover, $p$ is considered to be the deviation of the inverse of the maximum value of the strength measure from the inverse of the average value of the strength measure. In this sense of $p$, the index $p \sigma(\boldsymbol{u})$ in (6) refers to a normalized index for the deviation of the strength measure.

On the other hand, as a cost function that has a tradeoff relation with respect to $f_{0}$, we define the following constraint function for the mass:

$$
f_{1}(\phi)=\int_{\Omega(\phi)} \rho \mathrm{d} x-c_{1},
$$

where $\rho \in W^{1, \infty}\left(D_{0} ; \mathbb{R}\right)$ is the density, and $c_{1}$ is a positive constant such that there exists some $\phi \in \mathcal{D}$ that satisfies $f_{1}(\phi)<0$.

Using the cost functions, we construct the shape optimization problem as follows.

Problem 2 (Strength maximization problem)

Let $f_{0}(\boldsymbol{\phi}, \boldsymbol{u}, p)$ and $f_{1}(\boldsymbol{\phi})$ be defined in (5) and (7), 
respectively. Find $\phi$ such that

$$
\begin{aligned}
\min _{\boldsymbol{\phi} \in \mathcal{D}}\left\{f_{0}(\boldsymbol{\phi}, \boldsymbol{u}, p) \mid f_{1}(\boldsymbol{\phi})\right. & \leq 0, \\
\boldsymbol{u} & \in \mathcal{S}, \text { Problem } 1 \text { and }(6)\} .
\end{aligned}
$$

\section{Shape derivative of the cost functions}

Let $\varphi \in Y$ be the domain variation from $\phi$. We refer to the Fréchet derivatives of $f_{0}$ and $f_{1}$ with respect to arbitrary $\varphi \in Y$ as the shape derivatives, and we denote these derivatives as $f_{0}^{\prime}(\boldsymbol{\phi}, \boldsymbol{u}, p)\left[\boldsymbol{\varphi}, \boldsymbol{u}^{\prime}, p^{\prime}\right]$ and $f_{1}^{\prime}(\boldsymbol{\phi})[\boldsymbol{\varphi}]$, respectively, where $\boldsymbol{u}^{\prime}$ and $p^{\prime}$ denote the variations of $\boldsymbol{u}$ and $p$, respectively, caused by $\varphi$ that satisfy Problem 1 and (6).

The shape derivative of $f_{1}$ is obtained using the formula of the shape derivative for the domain integral [7] as

$$
f_{1}^{\prime}(\boldsymbol{\phi})[\boldsymbol{\varphi}]=\left\langle\boldsymbol{g}_{1}, \boldsymbol{\varphi}\right\rangle=\int_{\partial \Omega(\boldsymbol{\phi})} \rho \boldsymbol{\nu} \cdot \boldsymbol{\varphi} \mathrm{d} \gamma
$$

On the other hand, the shape derivative of $f_{0}$ is obtained as follows. Let

$$
\begin{aligned}
& \mathscr{L}_{0}\left(\boldsymbol{\phi}, \boldsymbol{u}, \boldsymbol{v}_{0}, p, q_{0}\right) \\
& =f_{0}(\boldsymbol{\phi}, \boldsymbol{u}, p)+q_{0}\left(p+\frac{1}{c_{0}}-\frac{\int_{\Omega(\phi)} \mathrm{d} x}{\int_{\Omega(\boldsymbol{\phi})} \sigma(\boldsymbol{u}) \mathrm{d} x}\right) \\
& \quad+\mathscr{L}_{\mathrm{M}}\left(\boldsymbol{\phi}, \boldsymbol{u}, \boldsymbol{v}_{0}\right)
\end{aligned}
$$

be the Lagrangian for $f_{0}$, where $\boldsymbol{v}_{0} \in \mathcal{S}$ and $q_{0} \in \mathbb{R}$ are introduced as Lagrange multipliers. The shape derivative of $\mathscr{L}_{0}\left(\boldsymbol{\phi}, \boldsymbol{u}, \boldsymbol{v}_{0}, p, q_{0}\right)$ is written as follows:

$$
\begin{aligned}
\mathscr{L}_{0}^{\prime}\left(\boldsymbol{\phi}, \boldsymbol{u}, \boldsymbol{v}_{0}, p, q_{0}\right)\left[\boldsymbol{\varphi}, \boldsymbol{u}^{\prime}, p^{\prime}\right] \\
=\mathscr{L}_{0 \boldsymbol{\phi}}\left(\boldsymbol{\phi}, \boldsymbol{u}, \boldsymbol{v}_{0}, p, q_{0}\right)[\boldsymbol{\varphi}]+\mathscr{L}_{0 \boldsymbol{u}}\left(\boldsymbol{\phi}, \boldsymbol{u}, \boldsymbol{v}_{0}, p, q_{0}\right)\left[\boldsymbol{u}^{\prime}\right] \\
\quad+\mathscr{L}_{0 \boldsymbol{v}_{0}}\left(\boldsymbol{\phi}, \boldsymbol{u}, \boldsymbol{v}_{0}, p, q_{0}\right)\left[\boldsymbol{v}_{0}^{\prime}\right]+\mathscr{L}_{0 p}\left(\boldsymbol{\phi}, \boldsymbol{u}, \boldsymbol{v}_{0}, p, q_{0}\right)\left[p^{\prime}\right] \\
\quad+\mathscr{L}_{0 q_{0}}\left(\boldsymbol{\phi}, \boldsymbol{u}, \boldsymbol{v}_{0}, p, q_{0}\right)\left[q_{0}^{\prime}\right] .
\end{aligned}
$$

Here, if $\boldsymbol{u}$ is the solution to Problem 1 and $p$ is determined by (6), then the third and fifth terms in the righthand side of (9) become 0 .

The fourth term in the right-hand side of (9) is calculated as

$$
\begin{aligned}
\mathscr{L}_{0 p}\left(\boldsymbol{\phi}, \boldsymbol{u}, \boldsymbol{v}_{0}, p, q_{0}\right)\left[p^{\prime}\right] \\
=\left(-\frac{1}{p^{2}} \ln \frac{\int_{\Omega(\boldsymbol{\phi})} \mathrm{e}^{p \sigma(\boldsymbol{u})} \mathrm{d} x}{\int_{\Omega(\boldsymbol{\phi})} \mathrm{d} x}-\frac{\int_{\Omega(\boldsymbol{\phi})} \mathrm{e}^{p \sigma(\boldsymbol{u})} \sigma(\boldsymbol{u}) \mathrm{d} x}{p \int_{\Omega(\boldsymbol{\phi})} \mathrm{e}^{p \sigma(\boldsymbol{u})} \mathrm{d} x}+q_{0}\right) p^{\prime} .
\end{aligned}
$$

Then, the fourth term becomes 0 , if $q_{0}$ is determined by

$$
q_{0}=\frac{1}{p^{2}} \ln \frac{\int_{\Omega(\phi)} \mathrm{e}^{p \sigma(\boldsymbol{u})} \mathrm{d} x}{\int_{\Omega(\phi)} \mathrm{d} x}+\frac{\int_{\Omega(\phi)} \mathrm{e}^{p \sigma(\boldsymbol{u})} \sigma(\boldsymbol{u}) \mathrm{d} x}{p \int_{\Omega(\phi)} \mathrm{e}^{p \sigma(\boldsymbol{u})} \mathrm{d} x} .
$$

Moreover, the second term in the right-hand side of (9) is calculated as

$$
\mathscr{L}_{0 \boldsymbol{u}}\left(\boldsymbol{\phi}, \boldsymbol{u}, \boldsymbol{v}_{0}, p, q_{0}\right)\left[\boldsymbol{u}^{\prime}\right]
$$

$$
\begin{aligned}
&= \frac{\int_{\Omega(\phi)} \mathrm{e}^{p \sigma(\boldsymbol{u})} \frac{\partial \sigma(\boldsymbol{u})}{\partial \boldsymbol{T}(\boldsymbol{u})} \cdot \boldsymbol{T}\left(\boldsymbol{u}^{\prime}\right) \mathrm{d} x}{p \int_{\Omega(\phi)} \mathrm{e}^{p \sigma(\boldsymbol{u})} \mathrm{d} x} \\
&+q_{0} \frac{\int_{\Omega(\phi)} \mathrm{d} x \int_{\Omega(\phi)} \mathrm{e}^{\sigma(\boldsymbol{u})} \frac{\partial \sigma(\boldsymbol{u})}{\partial \boldsymbol{T}(\boldsymbol{u})} \cdot \boldsymbol{T}\left(\boldsymbol{u}^{\prime}\right) \mathrm{d} x}{\left(\int_{\Omega(\phi)} \sigma(\boldsymbol{u}) \mathrm{d} x\right)^{2}} \\
&+\mathscr{L}_{\mathrm{M}}\left(\boldsymbol{\phi}, \boldsymbol{u}^{\prime}, \boldsymbol{v}_{0}\right) \\
&=\int_{\Omega(\boldsymbol{\phi})}\left(\boldsymbol{u}, p, q_{0}\right) \cdot \boldsymbol{T}\left(\boldsymbol{u}^{\prime}\right) \mathrm{d} x+\mathscr{L}_{\mathrm{M}}\left(\boldsymbol{\phi}, \boldsymbol{u}^{\prime}, \boldsymbol{v}_{0}\right) .
\end{aligned}
$$

Then, the second term becomes 0 if $\boldsymbol{v}_{0}$ is the solution to the following adjoint problem.

Problem 3 (Adjoint problem for $f_{0}$ ) Let $\boldsymbol{u}$ be the solution to Problem 1, and let $p$ and $q_{0}$ be given by (6) and (10), respectively. Find $\boldsymbol{v}_{0} \in \mathcal{S}$ such that

$$
\begin{aligned}
& -\nabla^{\mathrm{T}} \boldsymbol{T}\left(\boldsymbol{v}_{0}\right)=-\boldsymbol{\nabla}^{\mathrm{T}} \boldsymbol{\Sigma}\left(\boldsymbol{u}, p, q_{0}\right) \text { in } \Omega(\boldsymbol{\phi}), \\
& \boldsymbol{T}\left(\boldsymbol{v}_{0}\right) \boldsymbol{\nu}=\mathbf{0} \text { on } \Gamma_{p}^{-}(\boldsymbol{\phi}), \\
& \boldsymbol{T}\left(\boldsymbol{v}_{0}\right) \boldsymbol{\nu}=\mathbf{0} \text { on } \Gamma_{\mathrm{N}}^{-}(\boldsymbol{\phi}) \backslash \bar{\Gamma}_{p}(\boldsymbol{\phi}), \\
& \boldsymbol{v}_{0}=\mathbf{0} \text { on } \Gamma_{\mathrm{D}}(\boldsymbol{\phi}),
\end{aligned}
$$

where $\boldsymbol{\Sigma}\left(\boldsymbol{u}, p, q_{0}\right)$ is defined in (11).

Considering the above conditions, if we use the solutions of $\boldsymbol{u}$ and $\boldsymbol{v}_{0}$ of Problem 1 and Problem 3, respectively, and $p$ and $q_{0}$ are determined by (6) and (10), respectively, then (9) becomes

$$
\begin{aligned}
\mathscr{L}_{0 \boldsymbol{\phi}}\left(\boldsymbol{\phi}, \boldsymbol{u}, \boldsymbol{v}_{0}, p, q_{0}\right)[\boldsymbol{\varphi}] & \\
=f_{0}^{\prime}(\boldsymbol{\phi}, \boldsymbol{u}, p)[\boldsymbol{\varphi}] & \\
= & \int_{\partial \Omega^{-}(\phi)} \zeta_{\partial \Omega} \boldsymbol{\nu} \cdot \boldsymbol{\varphi} \mathrm{d} \gamma+\int_{\Gamma_{\mathrm{D}}^{-}(\boldsymbol{\phi})} \zeta_{\mathrm{D}} \boldsymbol{\nu} \cdot \boldsymbol{\varphi} \mathrm{d} \gamma \\
& \quad+\int_{\Gamma_{\mathrm{N}}^{-}(\boldsymbol{\phi})}\left(\boldsymbol{\varphi} \cdot \boldsymbol{\nabla} \zeta_{\mathrm{N}}+\kappa \zeta_{\mathrm{N}}\right) \boldsymbol{\nu} \cdot \boldsymbol{\varphi} \mathrm{d} \gamma \\
& +\int_{\partial \Gamma_{\mathrm{N}}(\boldsymbol{\phi}) \cup \Theta(\phi)} \zeta_{\mathrm{N}} \boldsymbol{\tau} \cdot \boldsymbol{\varphi} \mathrm{d} \varsigma=\left\langle\boldsymbol{g}_{0}, \boldsymbol{\varphi}\right\rangle
\end{aligned}
$$

where

$$
\begin{gathered}
\zeta_{\partial \Omega}=\frac{-1}{p \int_{\Omega(\phi)} \mathrm{d} x}+\frac{\mathrm{e}^{p \sigma(\boldsymbol{u})}}{p \int_{\Omega(\phi)} \mathrm{e}^{p \sigma(\boldsymbol{u})} \mathrm{d} x} \\
\quad+\frac{q_{0} p \sigma(\boldsymbol{u})}{\int_{\Omega(\phi)} \mathrm{d} x}-\frac{q_{0} p \int_{\Omega(\phi)} \sigma(\boldsymbol{u}) \mathrm{d} x}{\left(\int_{\Omega(\phi)} \mathrm{d} x\right)^{2}} \\
\quad-\boldsymbol{T}(\boldsymbol{u}) \cdot \boldsymbol{E}\left(\boldsymbol{v}_{0}\right)+\boldsymbol{b} \cdot\left(\boldsymbol{u}+\boldsymbol{v}_{0}\right) \\
\zeta_{\mathrm{D}}=\boldsymbol{T}\left(\boldsymbol{u}-\boldsymbol{u}_{\mathrm{D}}\right) \cdot \boldsymbol{E}\left(\boldsymbol{v}_{0}\right)+\boldsymbol{T}\left(\boldsymbol{v}_{0}-\boldsymbol{u}_{\mathrm{D}}\right) \cdot \boldsymbol{E}(\boldsymbol{u}) \\
\zeta_{\mathrm{N}}=\boldsymbol{p} \cdot\left(\boldsymbol{u}+\boldsymbol{v}_{0}\right)
\end{gathered}
$$

in which $\tau$ denotes the outer unit tangent of $\Gamma_{\mathrm{N}}^{-}(\phi)$ for $d=2$ and the outer unit tangent of $\Gamma_{\mathrm{N}}^{-}(\phi)$ and the outer unit normal of $\partial \Gamma_{\mathrm{N}}(\phi) \cup \Theta(\phi)$ for $d=3$, and $\kappa=\nabla \cdot \boldsymbol{\nu}$. 


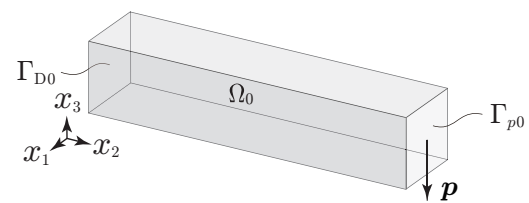

(a) Linear elastic problem (Problem 1)

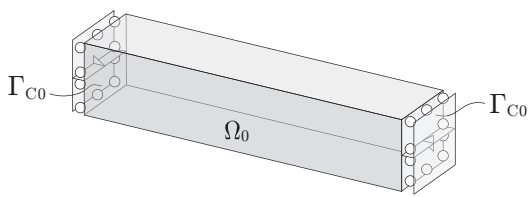

(b) Domain variation

Fig. 2. Boundary conditions in the cantilever problem.

\section{Solution}

The algorithm for solving Problem 2 based on the sequential quadratic programming is shown in previous papers $[5,6]$. In this algorithm, the $H^{1}$ gradient method is used for reshaping with shape derivatives $\boldsymbol{g}_{0}$ and $\boldsymbol{g}_{1}$ in (12) and (8), respectively.

\section{Numerical example}

We developed a program based on an algorithm using the finite element method. Using this program, we solved a strength maximization problem of the cantilever as shown in Fig. 2. We assumed $\Omega_{0}=(0,1) \times(0,5) \times(0,1)$, $\boldsymbol{p}=(0,0,-1)^{\mathrm{T}} \psi$ with basis function $\psi$ for the node at the center of $\Gamma_{p}(\phi)$, and $\boldsymbol{u}_{\mathrm{D}}=\mathbf{0}$ on $\Gamma_{\mathrm{D}}(\boldsymbol{\phi})$, as shown in Fig. 2(a). The stiffness $\boldsymbol{C}$ was constructed with a Young's modulus of $7.1 \times 10^{10}$ and a Poisson's ratio of 0.33 . In the domain variation, we assumed that the normal direction on $\Gamma_{\mathrm{C} 0}$ in Fig. 2(b), the vertical direction on the center lines of $\Gamma_{\mathrm{C} 0}$, and the horizontal direction at the center points of $\Gamma_{\mathrm{C} 0}$ were constrained. The finite element model of the cantilever was made with the P2 element.

The initial and optimized finite element models colored according to von Mises stress are shown in Fig. 3. The results indicate that the maximum value of the von Mises stress is reduced. The iteration histories of cost functions $f_{0}$ and $f_{1}$ normalized with the initial value $f_{\text {Oinit }}$ and $c_{1}$, respectively, are shown in Fig. 4 . In this figure, the plots labeled " $p$ in (6)" are the results obtained by the present method. Using the present method, $p$ was found to be 6.96 for the initial shape and 6.18 for the 40th iteration of reshaping. Compared with the shown results obtained using fixed values of $p$ of 5 and 40, the result obtained using the present method have good convergence properties.

\section{Acknowledgments}

The present study was supported by JSPS KAKENHI (20540113).

\section{References}

[1] G. Kreisselmeier and R. Steinhauser, Application of vector performance optimization to a robust control loop design for a fighter aircraft, Int. J. Control, 37 (1983), 251-284.

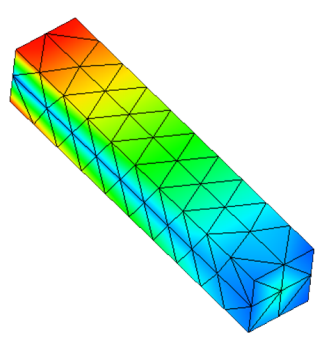

(a) Initial

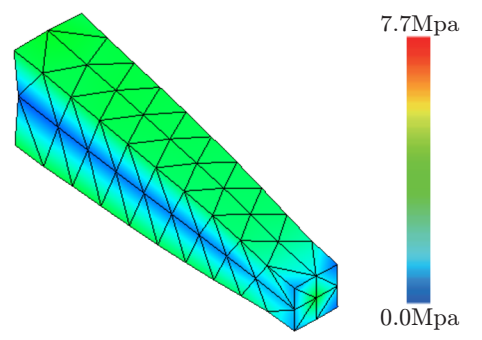

(b) Optimized
Fig. 3. Finite element models with meshes colored according to von Mises stress.

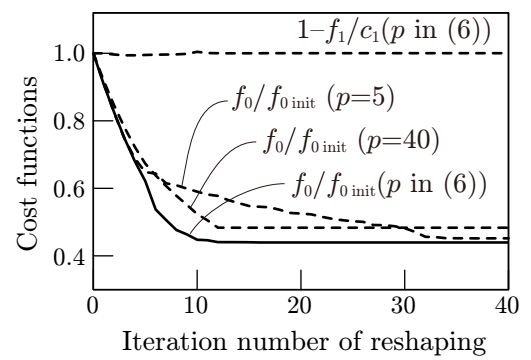

Fig. 4. Iteration histories of cost functions with respect to reshaping.

[2] M. Shimoda, H. Azegami and T. Sakurai, Numerical solution for min-max problems in shape optimization: minimum design of maximum stress and displacement, JSME Int. J. Ser. A, 41 (1998), 1-9.

[3] H. Azegami, Solution to domain optimization problems (in Japanese), Trans. JSME Ser. A, 60 (1994), 1479-1486.

[4] H. Azegami and K. Takeuchi, A smoothing method for shape optimization: traction method using the Robin condition, Int. J. Comput. Methods, 3 (2006), 21-33.

[5] H. Azegami, S. Fukumoto and T. Aoyama, Shape optimization of continua using NURBS as basis functions, Struct. Multidiscip. O., 47 (2013), 247-258.

[6] D. Murai and H. Azegami, Error analysis of H1 gradient method for shape-optimization problems of continua, JSIAM Letters, 3 (2013), 29-32.

[7] J. Sokolowski and J. P. Zolésio, Introduction to Shape Optimization: Shape Sensitivity Analysis, Springer-Verlag, New York, 1992. 